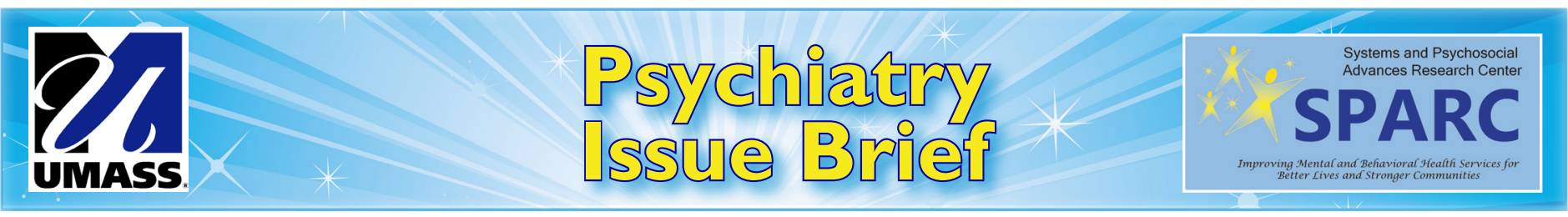

Vol 4 lss 5

Revised

Jan 2017
A Publication of the Systems and Psychosocial Advances Research Center

A Massachusetts Department of Mental Health Research Center of Excellence

\title{
Evidence Based Practices in Mental Health: Advantages, Disadvantages, and Research Considerations
}

Colleen E. McKay, MA, CAGS

$\mathrm{O}_{\mathrm{p}}^{\mathrm{n}}$ ne widely accepted definition of Evidence Based Practices (EBPs) is that they are interventions for which scientific evidence consistently shows that the practice improves client outcomes. ${ }^{1}$ EBPs rely on the classifications of research studies and findings according to a variety of evidence. In general, the highest standard requires evidence from several Randomized Clinical Trials (RCTs) by multiple teams of investigators comparing the practice to alternative practices or to no intervention. EBPs provide evidence of effective treatments or services based on rigorous research approaches that include randomization, control groups, studies with matched participants, blinding of service providers (so they are unaware of study participants or the treatment each individual receives), statistical analysis (often meta-analysis), and drawing accurate conclusions from study results. ${ }^{2}$

A panel convened by the Robert Wood Johnson Foundation in 1998 identified six program models providing services to adults with serious mental illness (SMI) as EBPs. The six models are illness management and recovery, medication management, assertive community treatment, family psycho-education, supported employment, and integrated dual diagnosis treatment. ${ }^{3}$ Today, a wide range of models serving persons diagnosed with SMI that produce beneficial outcomes have accrued varying levels of evidentiary support from RCTs, quasi-experimental research designs, and other systematic research evaluation methods. These models are listed on a variety of EBP registries and databases on the internet including The Campbell Collaboration, The Cochrane Collaboration, and the Substance Abuse and Mental Health Services Administration's (SAMHSA's) National Registry of Evidence-based Programs and Practices (NREPP). Other practices have not been reviewed or summarized with the intensity and rigor or examined using research designs sufficient to label them EBPs despite the increase in internet EBP registries

\section{Advantages \& Disadvantages of Evidence Based Practices}

There are several advantages to identifying services as EBPs:

- EBPs identify effective interventions based on reviews of multiple rigorous studies rather than on subjective interpretations of the reviewer, clinician, or stakeholder(s);

- EBP services receive support from a broader research base that includes psychological and biological research, and sociological evidence from multiple studies;

- Identification of EBPs may allow funders to direct limited resources to programs and areas where they will have the greatest impact;

- Many EBPs have corresponding manuals and guidelines to assist with service implementation and fidelity to a particular model; and

- Some EBPs have support from systematic assessments of existing research studies (meta-analysis) which allow readers to draw conclusions from a body of research. Meta-analysis offers a consolidated quantitative review to evaluate the results from multiple studies.

While identifying services as EBPs has advantages, over reliance on EBPs also has some distinct disadvantages:

- Limiting services to only EBPs may fail to incorporate models supported by consumer advocates or persons with lived experience. ${ }^{4}$ These models may not be identified as EBPs or offered within a system of care because of a lack of empirical research, not because they lack effectiveness; 
- $\quad$ Restricting EBP research to RCTs may limit participation to individuals with specific diagnostic criteria in order to enhance effect sizes. While serving the immediate research needs, addressing the effectiveness for the broader population is beyond the scope of most RCTs; ${ }^{5}$

- Most EPBs have not been developed and tested for specific cultural groups. Systematic methods for implementing EBPs to address culture-specific issues are vital;

- Many existing services or programs have yet to be included in research, making it impossible to know which have the best outcomes. We have no scientific way of knowing how these programs compare to EBPs unless we include these programs in our research;

- Evidence that examines the long-term effects of some EBPs does not exist. A service proven effective at one point in time does not mean that particular service will provide long-term lasting benefits; and

- Issues of adequate funding and fidelity to a particular model may affect the generalizability of study findings. Funding for services is often inadequate making full implementation of EBPs difficult, which could compromise fidelity to a particular EBP model.

\section{Recommendations for Policy Makers, Researchers, \& Service Providers}

1. Consider a range of evidence as an alternative means of classifying and assessing EBPs. The extent to which there is evidence from quasi-experimental designs, qualitative studies, case studies, or testimony from program participants may be useful in evaluating services. Consider designing studies that examine a wider variety of programs and services located in prevalent systems of care to build the evidence base for programs and services that need to be scrutinized using rigorous research methods.

2. Devote resources to examine services that have not undergone rigorous scrutiny to determine their effectiveness. In order to make viable comparisons between different program models we must provide tests that allow us to draw fair conclusions. ${ }^{6}$ Comprehensive research examining a wider variety of existing and innovative services, particularly those with fidelity to their respective models, will increase the quantity and quality of the evidence base; and

3. Consider the needs of the "consumer voice" or voices of persons with lived experience. There is considerable support for a variety of services from consumer advocates and the recovery movement., ${ }^{4}$ Studies or reviews of the effectiveness of mental health services readily adopted by consumers and stakeholders and EBPs may be beneficial.

\section{An Example of an EBP Review Conducted Within SPARC}

The Program for Clubhouse Research at UMass Medical School's SPARC led a systematic review of the evidence for the Clubhouse Model of Psychosocial Rehabilitation. The Clubhouse Model was reviewed and included in the list of programs on SAMHSA's NREPP in 2010. Clubhouses strive to help members (adults and young adults living with SMI) participate in mainstream employment, educational opportunities, community-based housing, and health promotion activities, in order to reduce hospitalizations or involvement with the criminal justice system, and improve satisfaction, social relationships, and quality of life.

The review conducted within SPARC targeted research on outcomes in a variety of domains associated with the Clubhouse Model including employment, education, social relationships, quality of life, health promotion activities, and hospitalizations. ${ }^{8}$ This review indicated higher levels of evidence for the Clubhouse Model including at least one RCT in the domains of hospitalization, quality of life, or employment.

Evidence for other domains appeared promising, as there was evidence from multiple observational studies that suggested the Clubhouse Model had a positive impact. However, there is a need for additional studies using rigorous methods including RCTs, studies with matched participants, or observational studies to evaluate programs with fidelity to the Clubhouse Model. Studies that examine the Clubhouse Model and other established EBPs would be useful. Efforts such as these are important steps in examining services that would benefit from additional research and/or designated as an EBP. 


\section{References}

1. Drake, R. E., Goldman, H. H., Leff, H. S., Lehman, A. F., Dixon, L., Mueser, K. T., \& Torrey, W. C. (2001). Implementing evidence-based practices in routine mental health service settings. Psychiatric Services, 52, 179-182.

2. Bilsker, D., \& Goldner, E. M. (2000). Teaching evidence-based practice in mental health. Research on Social Work Practice, 10, 664-669.

3. Substance Abuse \& Mental Health Services Administration. Promoting Recovery with Proven Solutions. Retrieved from http://www.samhsa.gov/samhsa_news/ VolumeXI_2/article3.htm

4. Frese, F. J. III., Stanley, J., Kress, K., \& Vogel-Scibilia, S. (2001). Integrating evidence-based practices and the recovery model. Psychiatric Services, 52, $1462-1468$.

5. Repper, J., \& Brooker, C. (1998). Difficulties in the measurement of outcome in people who have serious mental health problems. Journal of Advanced Nursing, $27(1), 75-82$.

6. Wolff, N. (2000). Using randomized controlled trials to evaluate socially complex services: Problems, challenges, and recommendations. The Journal of Mental Health Policy and Economics, 3, 97-109.

7. Wells, K., Miranda, J., Bruce, M. L., Alegria, M., \& Wallerstein, N. (2004). Bridging community intervention and mental health services research. American Journal of Psychiatry, 161, 955-963.

8. McKay, C., Nugent, K. L., Johnsen, M., Eaton, W. W., \& Lidz, C. W. (2016). A systematic review of evidence for the clubhouse model of psychosocial rehabilitation. Administration and Policy in Mental Health and Mental Health Services Research. Retrieved from: http://link.springer.com/article/10.1007/s10488-016-0760-3 